\title{
Advanced and nano manufacturing research at NSF
}

\section{Khershed Cooper}

Khershed P. Cooper, "Advanced and nano manufacturing research at NSF," Proc. SPIE 10982, Micro- and Nanotechnology Sensors, Systems, and Applications XI, 1098213 (13 May 2019); doi: 10.1117/12.2520329

SPIE Event: SPIE Defense + Commercial Sensing, 2019, Baltimore, MD, United States 


\title{
Advanced and Nano Manufacturing Research at NSF
}

\author{
Khershed P. Cooper* \\ National Science Foundation, 2415 Eisenhower Ave., Alexandria, VA, USA 22314 \\ *khcooper@nsf.gov; 703-292-7017
}

\begin{abstract}
The U.S. National Science Foundation (NSF)'s 'new' Advanced Manufacturing (AM) program is an amalgam of previous programs that explored nano-scale, additive or subtractive manufacturing, manufacturing machines or materials engineering or cybermanufacturing. The AM program seeks new ideas in, across and outside these domain areas, especially manufacturing at different scales and their integration to generate complex systems. A key component of the AM program is nanomanufacturing and an important application area for nanomanufacturing is flexible hybrid electronics (FHE). This paper describes the AM program and achievements in nanomanufacturing research. Nanomanufacturing research efforts through the core program, solicitations and center-level are highlighted. Projects in FHE that address challenges in processes, such as printing, imprinting and self-assembly, in materials, such as inks and substrates, and in scale-up, e.g., wafer-scale or continuous roll-to-roll are described. The paper is an overview of activities in these areas and is meant to serve as a resource for information and collaboration.
\end{abstract}

Keywords: Advanced manufacturing, nanomanufacturing, flexible hybrid electronics

\section{INTRODUCTION}

The mission of NSF is to promote progress in science and engineering and to advance national health, prosperity and welfare. NSF supports all fundamental science and engineering fields, except medical sciences. It ensures that research is integrated with education so that today's revolutionary work will also be training tomorrow's top scientists and engineers. Research programs at NSF are designed to fulfill these goals. The Advanced Manufacturing program is one whose goal is to support basic research activities that would result in new knowledge and education in materials engineering, processing and manufacturing.

\section{ADVANCED MANUFACTURING}

\subsection{The transformation of manufacturing}

Manufacturing has transformed over the decades. The transformation has been on several fronts-from mechanical systems to electronic systems, from analog to digital, from domestic production to off-shoring, from Industry 1.0 to Industry 4.0. New concepts are being developed-Factory of the Future, Distributed Manufacturing, Smart Manufacturing. To meet these challenges, what is needed are ways to cultivate the knowledge base for new manufacturing, develop new manufacturing concepts, create infrastructure for new production, acquire new skills. A paradigm shift in manufacturing is sought.

Advanced Manufacturing is the application of innovative technologies to accelerate product development, customize products, increase production efficiency, increase productivity and reduce cost. The need is for a rapid transfer of science and technology into manufacturing processes and products. The demands are that products are becoming technologically complex, require high level of design, need to be new, exciting, reliable, environmentally friendly and affordable. Additionally, products are needed to solve societal problems. To meet these demands, advances in process technologies such as CAD/CAM, precision, robotics, automation, intelligent systems, control systems, IT and modeling and simulation are leveraged.

Why Advanced Manufacturing? Manufacturing innovation is needed from early-stage research through production. The gap is in moving from research to prototype to product. This requires solving engineering design problems, overcoming cost burdens, inventing new production processes, creating efficient systems and educating a workforce for new manufacturing. To close this gap, a close connection between research, design and production phases is required by having constant feedback between production phase and research, and by tying innovation to the understanding of manufacturing processes.

Micro- and Nanotechnology Sensors, Systems, and Applications XI, edited by Thomas George,

M. Saif Islam, Proc. of SPIE Vol. 10982, 1098213 - (c) 2019 SPIE

CCC code: $0277-786 \mathrm{X} / 19 / \$ 18 \cdot$ doi: $10.1117 / 12.2520329$ 
To transform production, new manufacturing paradigms need cultivation. These are concepts such as network-centric production, i.e., embedding IT throughout the manufacturing value chain, mass customization, i.e., manufacturing based on customer's specification, nanomanufacturing, i.e., embedding nano-features to enhance product performance, and smart or energy efficient manufacturing. NSF's Advanced Manufacturing program supports research to meet these challenges and leads the transformation in manufacturing.

\subsection{NSF's Advanced Manufacturing program}

The goal of NSF's Advanced Manufacturing (AM) program is to support fundamental research to build a knowledge base to advance manufacturing science. Until mid-2018, four independent programs made up NSF's advanced manufacturing activities. (1) Manufacturing Machines and Equipment (MME), which advanced the development of new and/or improved manufacturing machines and equipment and optimization of their use. (2) Materials Engineering and Processing (MEP), whose goal was to discover mechanisms of process-structure-property-performance relationships in material systems driven by end-use application. (3) Nanomanufacturing (NM), which advanced the fundamental principles for nano-scale manufacturing processes that enable novel materials, structures, devices, and systems. (4) Cybermanufacturing Systems (CM), whose goal was to accelerate the creation of an interoperable, cross-process manufacturing service layer, built upon app-based infrastructure for manufacturing processes.

The Advanced Manufacturing program ${ }^{1}$ has several goals. (1) Fundamental research to revitalize American manufacturing, grow national prosperity and workforce, and reshape the Nation's strategic industries. (2) Multidisciplinary research to accelerate advances in manufacturing technologies that fundamentally alter and transform manufacturing capabilities, methods and practices. (3) Research that crosses and transcends domain boundaries in the 'old' CM, MME, MEP and NM areas. (4) Convergent research that brings manufacturing to new application areas by incorporating challenges and approaches outside the customary manufacturing portfolio. Furthermore, potential research areas in advanced manufacturing are identified ${ }^{2}$. They are manufacturing at all length scales, from nano to macro, integration of machine learning, network-accessed manufacturing services, manufacturing with extremes of current conditions and manufacturing materials and systems for extreme environments. While nanomanufacturing is not explicitly mentioned in the AM program descriptions, it is firmly entrenched in the advanced manufacturing lexicon.

\section{NANOMANUFACTURING}

Tremendous progress has been made in nano science, engineering and technology. New phenomena, such as quantum effects, have been studied. New materials and structures that, for example, stronger and lighter, have been made. New processes, such as self-assembly, have been discovered. New advances in metrology and standards have been made. New devices and systems, e.g., transistors, memory, have been built. For example, steady progress has been made in support of Moore's Law. Nanotechnology has impacted all industrial sectors-power and energy, electronics and communication, chemical and environmental, biomedical and pharmaceutical, and food. Societal benefits are plenty. However, the nano-scale poses several manufacturing challenges. These are process controllability, reproducibility, reliability; production scalability, yield, efficiency, safety, affordability; product manufacturability, quality and performance. Appropriate metrics, such as, precision of placement, feature size and resolution, overlay registration, nanostructure density, complexity and rate of formation, are needed for each nano-scale process. Practitioners may need to compromise on feature size and resolution as a function of processing rate and volume throughput. Strategies such as selection of materials and unit processes, fab integration and packaging need to be determined.

Nanomanufacturing is the fabrication of nano-scale building-blocks, nanomaterials and nanostructures, their assembly into higher-order structures, components and devices, and the integration of these into larger scale systems. It is the study of processes, techniques and methods to manipulate and control matter at the nanoscale in 1-, 2-, and 3dimensions, reproducibly. The nano-scale is usually defined as 1-100 nm, but it could be a greater nm dimension where a significant beneficial effect is expected. Processes can be bottom-up, i.e., self- and directed-assembly or top-down, such as, lithography, deposition, removal, or a combination of the two. Integration can be across scales (nano to meso), materials $(0 \mathrm{D}, 1 \mathrm{D}, 2 \mathrm{D})$, geometries, processes and functions. The nanomanufacturing value chain is represented as nanoscale building blocks $\rightarrow$ nanostructures and nano-assemblies $\rightarrow$ nanocomponents and nanodevices $\rightarrow$ nano-subsystems $\rightarrow$ nanosystems $\rightarrow$ products and research usually targets any of these stages where a critical roadblock is identified.

Nanomanufacturing research is needed to accelerate the transfer of lab-scale ideas into the marketplace, to meet the demands for increased complexity, increased performance, and new capabilities, to meet new nanotech-inspired grand challenges, to invent new production concepts and to determine process fundamentals, reliable techniques, design rules, and production metrics. Nanomanufacturing bridges the gap between nanoscience discoveries and nanotechnology products. 


\subsection{NSF's basic research investments in nanomanufacturing}

Very soon after the National Nanotechnology Initiative (NNI) was announced in 2000, NSF responded by initiating its Nanomanufacturing (NM) program, whose goal was to support fundamental research in novel nano-scale processes for large-scale manufacturing of nanomaterials and nanostructures. Following the President's Council of Advisors on Science and Technology (PCAST) recommendation in 2009 that Federal Agencies augment their investments in nanomanufacturing research, NSF announced the Scalable Nanomanufacturing (SNM) solicitation in 2011, which, in 2017, was revised to the Scalable Nanomanufacturing for Integrated Systems (SNM-IS) solicitation.

The goal of SNM was to study new approaches to overcome key scientific and technical barriers that prevent the manufacture of useful nano materials, structures, devices and systems at an industrially relevant scale, reliably, at low cost and within environmental, health and safety guidelines. The goal of SNM-IS was to study and formulate fundamental principles for scalable or customizable manufacturing and integration for nanotechnology-based integrated systems towards the eventual manufacture of useful nanotechnology products. The NM program and the SNM and SNM-IS solicitations are now part of the 'new' Advanced Manufacturing (AM) program. However, these initiatives generated a wealth of research results and breakthroughs, resulting in significant contributions to the fundamental knowledge base for nanomanufacturing and commercialization. These activities studied several research areas in materials, processes and applications as listed in Table 1.

Table 1. Nanomanufacturing research areas.

\section{MATERIALS}

- C-based: CNT, Graphene, Bucky-tape, CNT Fibers, Cellulosic

- OD: Nanoparticles, QDs, Core-shell, Janus, Hierarchical, Composite

- 1D: Nanowires, Nanopillars, Nanotubes, Nanofibers

- 2D: $\mathrm{MoS}_{2}, \mathrm{BN}, \mathrm{TMDs}$

- 3D: Nanoporous, Aerogels, MOFs

- Material Systems: Metals, Ceramics, Polymers, Organics, Biomaterials, Composites

\section{PROCESSES}

- Chemical/Thermal: Combustion, Plasma, Hydrothermal, Drawing, Etching

- Vapor-based: CVD, PVD, PECVD, Laser CVD, ALD, MLD

- Fluid/Solution-based: Coating, Casting, Colloids, Electrospray, Electrophoresis, Electrospinning, Electroetching, Microfluidics, Microreactors, Ink-jet Printing

- Lithography/Patterning: AFM/STM, DPN, NIL, PLD, FIB, EUV, EBL, BCP

- Assembly: Self, Directed (chemical, magnetic, acoustic), Molecular

- Mechanical: Exfoliation, Nanomachining, Ball-milling

- 3D Nanomanufacturing: 3D Nanoprinting, Holographic Lithography, MacEtch

- Bio-Inspired/Enabled: DNA, Virus, Protein, Peptides, Diatoms

\section{APPLICATIONS}

- Environmental: Water/Air Purification, Analytical Separation

- Chemical: Catalysis, Gas Storage

- Energy: Storage, Conversion, Harvesting, Batteries, Supercapacitors, PVs, Solar Cells, Fuel Cells

- $\quad$ Electronics: ICs, Flexible, Storage Memory, 3D Devices, TFTs, EM-Shielding

- Optoelectronics/Photonics: Imaging, Waveguides, Displays, Lighting, Metamaterials

- Sensors: Biological, Chemical, Multiplexed, Quantum

- $\quad$ Structural: High-Strength, Light-Weighting, Packaging

- Biomedical: Implants, Tissue Scaffolds, Diagnostics, Therapeutics, Drugs, Probes

- $\quad$ Sheets/Wires: Fibers, Cables, Filters, Membranes, Textiles, Paper, Fabric, Nonwovens 
To meet the scalability challenges, several scale-up approaches were investigated. Scale-up methods fell in approximately five categories. (1) Continuous roll-to-roll process with top-down and bottom-up methods, which included processes such as printing, imprinting, self-assembly, deposition, coating and lamination. (2) Parallel, largearea top-down or bottom-up methods, which included processes such as optical lithography, direct-write, self- and directed-assembly. (3) Parallel, large-area 3D nanofabrication, which included methods such as 2-photon polymerization, nanoimprinting and self-assembly and strain engineering. (4) Continuous or parallel reaction synthesis or fluidics, which included processes such as microreactor, microfluidic, hydrothermal, chemical, plasma, electrospray, electrospinning, and fiber-drawing. (5) Large-area bio-enabled nanofabrication, for example, templating using DNA.

\subsection{NSF's center-level research investments in nanomanufacturing}

NSF fielded four manufacturing Nano Science and Engineering Centers (NSECs), whose purpose was interdisciplinary research to understand nanoscale processes, develop tools for measurement and manufacturing at the nanoscale, develop concepts for high-rate synthesis and processing of nanostructures and nanosystems, and scale-up of nanoscale processing methods. While all NSECs are now graduated, they generated significant knowledge in nanomanufacturing tools and platforms. The Scalable and Integrated Nano Manufacturing (SINAM) ${ }^{3}$ center developed plasmonic nanolithography. Nano Chemical-Electrical-Mechanical Manufacturing Systems (Nano-CEMMS) ${ }^{4}$ center developed nanofluidics, electrohydrodynamic-jet and superionic solid-state stamping (S4) methods. The Center for High-Rate Nanomanufacturing $(\mathrm{CHN})^{5}$ has marketed its offset-printing system. The Center for Hierarchical Manufacturing $(\mathrm{CHM})^{6}$ developed a roll-to-roll nanomanufacturing system that uses directed self-assembly and nanoimprint lithography. Besides developing nanomanufacturing tools and platforms, which could be used for future research and prototyping, these centers have been very productive through technical papers, patents, and small business spin-offs. Many of them became partners in the Manufacturing USA Innovation Institutes, especially, NextFlex.

Additionally, one of NSF's Nanosystems Engineering Research Centers (NERCs), Nanomanufacturing Systems for Mobile Computing and Energy Technologies or NASCENT ${ }^{7}$ is developing a nanodevices manufacturability fabrication (nm-FAB) facility, which consists of a roll-to-roll (R2R) platform and a flexible wafer-scale (WS Flex) platform. The $\mathrm{nm}-\mathrm{FAB}$ nanosystem is based on enabling technologies, which are based on fundamental nanomanufacturing knowledge. NASCENT is developing several unit processes for the nm-FAB facility. Nanomaterials deposition, nanoshape imprinting, graphene transfer, nanoscale encapsulation and high-speed process and functional metrology in conjunction with existing metal/dielectric deposition and plasma/wet etch technologies for its roll-to-roll platform. Bulk crystalline exfoliation, nanoshape imprinting, process and functional metrology and multi-scale modeling along with existing nanomaterial ink-jet printing, plasma/wet etch and metal/dielectric deposition technologies.

Many of the basic and center-level research are applicable to the field of flexible hybrid electronics.

\section{FLEXIBLE HYBRID ELECTRONICS}

\subsection{NSF nanomanufacturing research in stretchable technologies and flexible hybrid electronics}

The theme of this session, Organic-Inorganic Stretchable Technologies, is a sub-topic of the Micro- and Nanotechnology Sensors, Systems and Applications XI Symposium. Stretchable, foldable, rollable and bendable electronic technologies can be together fall under the flexible hybrid electronics (FHE) rubric.

Several NM, SNM and SNM-IS projects are addressing challenges in FHE. Tables 2 and 3 list SNM and SNM-IS projects, respectively, in fundamental research in FHE. They give the project title, the name of the principal investigator, institution and a brief description of the research. The research involves different materials and novel processing approaches towards fabricating structures, components and devices on flexible substrates with scalability and high throughput. Research efforts are also in coming up with scalable or customizable integrated systems, paying particular attention to integration strategies.

Most of the manufacturing NSECs have developed nanomanufacturing tools which are particularly applicable to FHE technologies. A key application is human health monitoring, for which flexible sensors and actuators are being developed. The NASCENT NERC, with its focus on mobile devices, is especially positioned to meet FHE roadblocks. 
Table 2. SNM projects in FHE.

\section{SNM PROJECTS}

- Development of Ink-Jet Based Low Cost Roll-to-Roll Nanopatterning (i-R2R Nano) with Demonstration in Thin Film Photovoltaics - S. V. Sreenivasan, University of Texas at Austin

Roll-to-roll, nanoimprint patterning and block copolymer self-assembly of amorphous-Si for thin-film PVs

- Continuous and Large Scale Nanomanufacturing of Graphene and Carbon Nanotube Materials - Lingjie Guo, University of Michigan Ann Arbor

Continuous CVD synthesis on flexible substrates, integration of patterning and surface modification using CNTs and graphene for hybrid PVs, metamaterials, high-surface-area filters

- $\quad$ Roll-to-Roll Atomic/Molecular Layer Deposition - Yung-Cheng Lee, University of Colorado at Boulder

Roll-to-roll, atomic and molecular layer deposition with process control of conformal and ultrathin inorganic and organic films for gas diffusion-barrier coatings and coatings for $\mathrm{Li}$ ion battery electrodes

- A Versatile Microplasma-based Patterning Technology for Large-Scale, High Throughput Nanomanufacturing - Christian Zorman, Case Western University Roll-to-roll, microplasma-based direct-write fabrication of sub-100 nm patterns of metallic and metal oxide structures for nanoscale functional devices on flexible substrates

- Integration of Organic Solar Cells with Engineered Nanostructures for Scalable Manufacturing of Energy Conversion and Storage Devices-Wei You, University of North Carolina-Chapel Hill

Low-temperature, high-throughput wet coating processes to produce polymer solar cells/nanowire electrodes and supercapacitors for energy conversion and storage

- Scalable Volume-Nanomanufacturing of Unique Nanoparticles by Nanotemplated Printing, Deposition, and Exfoliation for Energy and Bio/Chemical Sensing - Stephen Chou, Princeton University

Nano-templated printing, deposition and exfoliation of nanoparticles with unique shape, structure and composition for bio-chemical sensing, solid state lighting, displays, batteries and fuel cell applications

- Scalable Nanomanufacturing of Metasurfaces \& Plasmonic Opto-Mechanical Systems - Regina Ragan, University of California-Irvine

Directed self-assembly of colloidal nanoparticles and electrospin direct writing of nanowires to produce nanoarchitectures with sub-wavelength metallic building blocks for functional devices for sensing, energy, imaging and light-guiding

- Physics Guided Innovation of Integrated Flash-light-sintering, Continuous Nanomaterial Synthesis and Roll-to-roll Deposition Processes - Chih-hung Chang, Oregon State University

Microreactor-assisted nanoparticle synthesis, roll-to-roll nanoparticle-ink deposition and flash-light sintering of semiconductor inks and patterned thin-films for devices on largearea flexible substrates

- Modulation of Surface Topography for Scalable Contact Printing - Mitchell Anthamatten, University of Rochester

Additive contact printing with shape memory polymer stamp having responsive topography of patterned organic and inorganic thin films and their multilayered stacks for clean, defect-free and high-resolution organic LED arrays

- Roll-to-Roll Nanoimprinting to Manufacture Large Area Metasurfaces for

Photonics and Optoelectronics - James Good, Oklahoma State University Multilayer devices over large areas through repetitive coating and UV-cured resin nanoimprinting on a moving substrate

- High-Throughput Scalable Manufacturing of High-Performance Organic Devices - Adam Moule, University of California at Davis Patterning technology for the design of printed organic semiconductor-based electronic devices, such as active matrix arrays of thin film transistors (TFTs) using the dopantinduced solubility control (DISC) method

- Scalable Surface Corrugation of Silicon Surfaces for Enhanced Light Trapping in Solar Cells - Sang Eon Han, University of New Mexico

Phase-mask-based interference lithography (PMBIL) to create light trapping features on thin-silicon substrates 
Table 3. SNM-IS projects in FHE.

\section{SNM-IS PROJECTS}

- Additive Nanomanufacturing of Integrated Systems for Personalized Health

Monitoring - Neil Dasgupta, University of Michigan Ann Arbor

Manufacturing methodology combining ALD with e-jet printing to directly print functional nanomaterials with unparalleled spatial resolution in 3D towards additive manufacturing of customizable integrated nanosystems onto non-planar, flexible surfaces for personalized health-monitoring

- $\quad$ Printing and Integration of Metal Nanowires and Organic Semiconductors for Large-Area Stretchable Electronics and Sensors - Yong Zhu, North Carolina State University

Investigate a printing-based scalable manufacturing approach to fabricate large-area, highresolution, stretchable pressure sensor arrays for electronic-skin (e-skin) by heterogeneously integrating Ag nanowire conductors, organic semiconductors, and elastomers

- Scalable Nanomanufacturing of Graphene-based Real-time Water Sensors through Customized Inkjet Printing - Deyang Qu, University of WisconsinMilwaukee

Tailor inkjet printing as a platform for scalable nanomanufacturing of low-cost, disposable, graphene-based sensor systems for water contaminants with a unit cost of several cents

- Manufacturing Autonomy for Directed Evolution of Materials (MADEMaterials) for Robust, Scalable Nanomanufacturing - David Hoelzle, Ohio State

Framework for autonomous material synthesis via directed evolution, enabling scale-up of the manufacture of complex nanomaterials in the face of process uncertainty

\subsection{NSF and Manufacturing USA}

To address industrial challenges, NSF is partnering with the Manufacturing USA Innovation Institutes in a unique way. The Advanced Manufacturing program issued a call for collaborative proposals to address basic research enabling innovation at Manufacturing USA Institutes ${ }^{8}$. The goals are several. Encourage proposals that address critical fundamental research needs in advanced manufacturing in one or more of the Manufacturing USA Institutes' focus areas. Resulting knowledge can, in turn, enable new technologies that feed into the innovation pipelines of one or more of the Manufacturing USA Institutes. Encourage proposals that include a collaboration with a Manufacturing USA Institute and leverage the facilities, infrastructure and member companies of that Institute. The argument is that if the Manufacturing USA research efforts, which are in the TRL 4-7 range, meet with scientific roadblocks that require fundamental understanding, then the institute can partner with a NSF investigator and submit a proposal to solve the problem.

In response to the NSF Manufacturing USA initiative and to address FHE challenges, NSF and NextFlex ${ }^{9}$, the FHE manufacturing innovation institute, organized a workshop ${ }^{10}$ in Accelerating Innovative Manufacturing Technologies for FHE in August 2018. At this workshop NSF principal investigators and NextFlex researchers met and discussed critical gaps in FHE such as materials, integration, power, reliability, and manufacturing and metrology tools. It is expected that such joint research efforts will benefit both NSF researchers and NextFlex partners.

\section{SUMMARY}

This article is a review of NSF's research efforts in nanomanufacturing, both at the individual project level and at the center scale. It discusses how nanomanufacturing is positioned within the 'new' advanced manufacturing program. It highlights projects in support of flexible hybrid electronics and stretchable electronics technologies. This article should be considered a resource for information and possible collaboration by its readers. 


\section{REFERENCES}

[1] Division of Civil, Mechanical and Manufacturing Innovation: Advanced (AM) Program, PD 19-088Y: https://www.nsf.gov/funding/pgm_summ.jsp?pims_id=505572.

[2] Dear Colleague Letter: Transforming the CMMI Advanced Manufacturing Core Programs to Revitalize the Nation's Strategic Industries, NSF 18-091: https://www.nsf.gov/pubs/2018/nsf18091/nsf18091.jsp.

[3] Center for Scalable and Integrated Nanomanufacturing (SINAM), Zhang, Xiang, University of California Berkeley, http://grantome.com/grant/NSF/CMMI-0751621.

[4] Center for Nanoscale Chemical-Electrical-Mechanical Manufacturing Systems (Nano-CEMMS), King, William, University of Illinois at Urbana-Champaign, https://mechanical.illinois.edu/research/affiliated-centers/centernanoscale-chemical-electrical-mechanical-manufacturing-systems.

[5] Center for High-rate Nanomanufacturing (CHN), Busnaina, Ahmed, Northeastern University, https://www.nanomanufacturing.us/.

[6] Center for Hierarchical Manufacturing (CHM), Watkins, James, University of Massachusetts, http://chm.pse.umass.edu/.

[7] Nanomanufacturing Systems for Mobile Computing and Mobile Energy Technologies (NASCENT), S. V. Sreenivasan, University of Texas at Austin, https://nascent.utexas.edu/.

[8] Dear Colleague Letter: Advanced Manufacturing Research to Address Basic Research Enabling Innovation at Manufacturing USA Institute, 17-030: https://www.nsf.gov/pubs/2017/nsf17030/nsf17030.jsp.

[9] NextFlex: America's Flexible Hybrid Electronics Manufacturing Institute, Thompson, Malcolm, Director, https://www.nextflex.us/.

[10] Workshop: Accelerating Innovative Manufacturing Technology For Flexible Hybrid Electronics, Aug 6-7, 2018, NextFlex Headquarters, San Jose, CA, https://www.nextflex.us/news-events/events/archive/workshop-acceleratinginnovative-mfg-tech/. 